\title{
Análise comparativa de três sistemas embarcados que auxiliam o controle da glicemia em diabéticos
}

\author{
Maurício B. Costa ${ }^{1}$, Adonias C. de Oliveira ${ }^{2}$, F. Luciano C. Martins Júnior ${ }^{1}$ \\ ${ }^{1}$ Tecnologia em Telemática - Instituto Federal de Educação, Ciência e Tecnologia do \\ Ceará (IFCE) Campus Tauá \\ Tauá - CE - Brasil \\ ${ }^{2}$ Ciência da Computação - Instituto Federal de Educação, Ciência e Tecnologia do \\ Ceará (IFCE) Campus Tianguá \\ Tianguá - CE - Brasil \\ mauriciocosta.cdd@gmail.com, adonias.oliveira@ifce.edu.br, \\ luciano.martinsdifce.edu.br
}

\begin{abstract}
Generally, diabetic patients are advised to check their blood glucose level 5 to 7 times a day. Several branches of computing and electronics have contributed to the development of methods and systems that efficiently assist at low cost the diagnosis, monitoring and control of the disease. In this context, the present work analyzes three embedded prototypes that can serve as a reference for building a system with lower costs, low energy consumption and good measurement accuracy, determining ideal for daily use in chronic disease.
\end{abstract}

Resumo. Geralmente, os pacientes diabéticos são aconselhados a verificar seu nivel de glicose no sangue 5 a 7 vezes por dia. Vários ramos da computação e eletrônica têm contribuído com desenvolvimento de métodos e sistemas que auxiliam eficientemente à baixo custo o diagnóstico, monitoramento e controle da doença. Neste contexto, o presente trabalho analisa três protótipos embarcados que podem servir como referencial para construção de um sistema de menor custos, de baixo consumo de energia e boa precisão na medição determinando ideal para o uso diário a doença crônica.

\section{Introdução}

A diabetes também conhecida como diabetes mellitus, doença crônica global, faz com que o indivíduo tenha excesso nos níveis de açúcar no sangue muito acima do esperado. A aglomeração de glicose no sangue é chamada de glicemia. Quando o pâncreas produz pouca ou nenhuma insulina ocorre a elevação da glicose no sangue que resulta nesse distúrbio.

$\mathrm{Na}$ área da Computação, muitas tecnologias têm sido desenvolvidas para pessoas que necessitam de auxílio no tratamento de uma determinada doença. No caso da diabetes pode ser citado alguns dispositivos construídos para controle de glicemia, por exemplo a bomba de infusão de insulina, que é um sistema de infusão contínua (SIC) caracterizado por simular o que ocorre no organismo dos indivíduos que não tem diabetes. Durante 24 horas esse dispositivo libera insulina para regular o nível de glicose a valores normais entre as refeições e liberar o remédio nos horários de alimentação [SDB 2015]. 
Também podem ser encontrados diversos aplicativos de aparelhos móveis do chamado "novo ecossistema digital de ferramentas direcionado por dados" que podem direcionar o indivíduo e os médicos especializados a gerenciar o melhor tratamento a diabetes [Heintzman 2015].

Diante isso, o presente artigo apresenta uma análise comparativa sobre sistemas microcontrolados aplicados ao monitoramento e/ou controle do nível de glicemia referenciando três trabalhos acadêmicos.

\section{Fundamentação Teórica}

Sistema embarcado, também conhecidos como sistemas embutidos, é qualquer equipamento que, incluindo um computador programável, mas em modo geral, não é um computador em si. Com potencial de grandes aplicações de diversos tipos otimizados em projeto [Wolf 2012]. Pode ser projetado utilizando um ou mais microcontroladores e executam tarefas específicas de acordo com sua documentação de requisitos [Oliveira Junior and Duarte 2011].

Uma unidade microcontroladora, do inglês Microcontroller unit (MCU), ou microcontrolador, é simplesmente um computador em um único chip contendo vários circuitos integrados, a saber, processador, memória, periféricos de entrada e saída, temporizadores, dispositivos de comunicação serial e outros componentes de hardware As suas principais vantagens são o baixo custo de aquisição e o uso em Sistemas Embarcados, que são envolvidos nos equipamentos eletrônicos [Penido and Trindade 2013].

Sistemas embarcados baseados em microntroladores são desenvolvidos para diversas aplicações como na construção de bengala e capacete ultrassônico para detecção de obstáculos para pessoas com deficiência visual [Chaves et al. 2018].

\section{Projetos desenvolvidos para monitoramento com sistemas microcontrolados.}

A busca dos trabalhos foi realizada via Google Acadêmico usando a string "microcontroller diabetes". Dentre os diversos trabalhos retornados foi selecionado o artigo de [Latha et al. 2012], porque trata sobre um projeto e desenvolvimento de um sistema embarcado baseado no microcontrolador PIC18F4520 que mede glicose no sangue. Foi escolhido o artigo de [Meshram et al. 2015], visto que utiliza o método semiinvasivo para medição de glicose no sangue. O artigo de [Silva et al. 2012] foi selecionado por ser integrado ao sistema Ambient Assisted Living sugerindo uma conexão wireless.

\subsection{Design and Development of a Microcontroller Based System for The Measurement of Blood Glucose [Latha, Murthy, and Sunitha 2012]}

Esse trabalho propõe um projeto e desenvolvimento um sistema baseado com uma MCU para medir glicose no sangue de pessoas com diabetes, através de um pequeno furo com agulha descartável para retirada de sangue do usuário. 


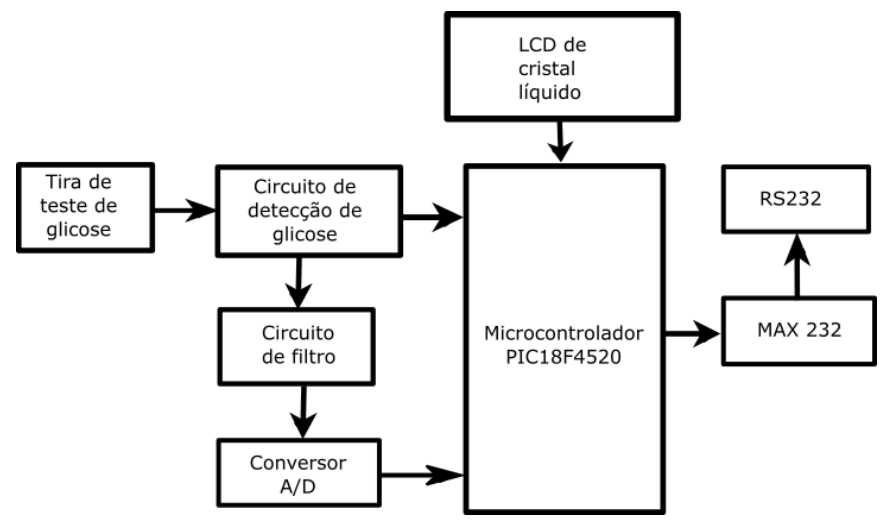

Figura 1 - Diagrama de Blocos de Projeto e Desenvolvimento de Sistema Baseado em Microcontrolador para a medição de Glicose no sangue.

A Figura 1 apresenta o diagrama de blocos de projeto e desenvolvimento de sistema baseado em microcontrolador para a medição de glicose no sangue. Como pode ser visto, ele é interligado a um display LCD, o qual mostra o resultado da operação realizada pelo PIC18F4520, através de uma fita de teste de glicemia conectada a um circuito de detecção de glicose, passando por um circuito de filtro e pelo conversor Analógico/Digital que converte o sinal filtrado para o microcontrolador.

\subsection{Microcontroller Based Instrument for Semi-Invasive Blood Glucose Measurement [Meshram and Dahikar 2015]}

O principal objetivo desse trabalho é a determinação da glicose no sangue e o conceito de sistema de medição de forma não invasiva, baseada em infravermelho em conjunto com o dispositivo.

Com a tecnologia de modem GSM o dispositivo aceita qualquer cartão SIM de operadora de rede GSM é possível atuar como um telefone celular com seu próprio número de telefone exclusivo, ou seja, enviar e receber SMS ou fazer/receber chamadas de voz. De acordo com [Meshram et al. 2015], o sistema apresenta a vantagem do uso do modem com a porta RS232 para comunicação e desenvolvimento de aplicativos que podem ser agregados à plataforma. Na Figura 2 é mostrado o esquema feito para a comunicação do modem GSM e a MCU com a utilização de um circuito integrado MAX232.

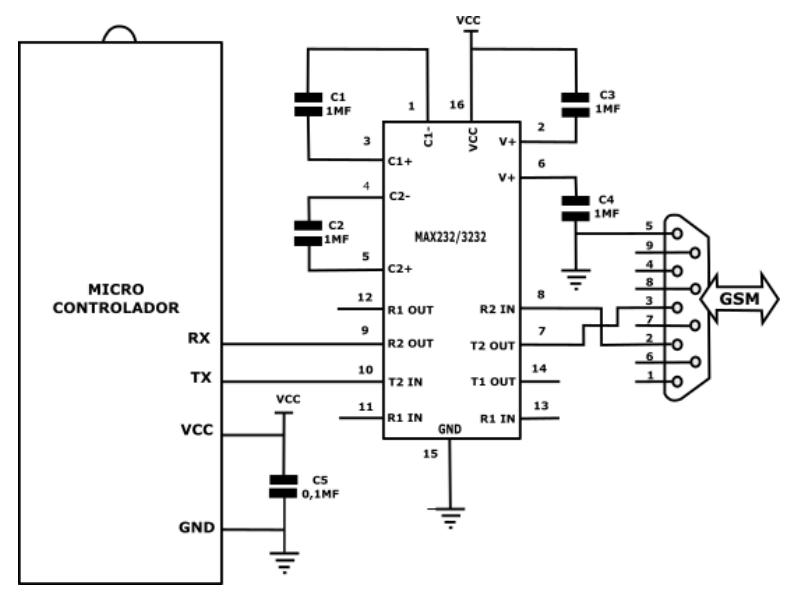

Figura 2 - Comunicação com GSM e microcontrolador através de MAX 232. 
Ele também pode ser usado no modo GPRS para se conectar à Internet e fazer muitos aplicativos para registro e controle de dados. Nesse modo pode se conectar a qualquer servidor de arquivos remoto e fazer upload de arquivos para o registro de dados.

\subsection{A bluetooth approach to diabetes sensing on Ambient Assisted Living systems} [Silva, Martins and Soares 2012].

O objetivo desse sistema foi reunir informações do sistema de monitoramento de glicose proposto por [Leal et al. 2011], que se conecta com o sistema Ambient Assisted Living (ALL). É composto por TMS37157, um dispositivo de baixa frequência, um microcontrolador CC2540, com solução Bluetooth integrado no chip, além de incluir os componentes Balun-Low Pass Filter 2450BM15A0002, que permite a conexão fácil da antena, e o TPS62730 que alimenta o sensor.

Conforme ilustra a Figura 3, o sistema é composto por dispositivo de medição de glicose que pode ser ligado a qualquer tecnologia que contenha comunicação bluetooth como um smartwatch (relógio inteligente), smartphone e o computador com o Ambient Assisted Living através da conexão ao adaptador bluetooth. Com essas tecnologias é possível apresentar os dados de glicemia em tempo real obtidos pelo dispositivo transmissor junto ao corpo.

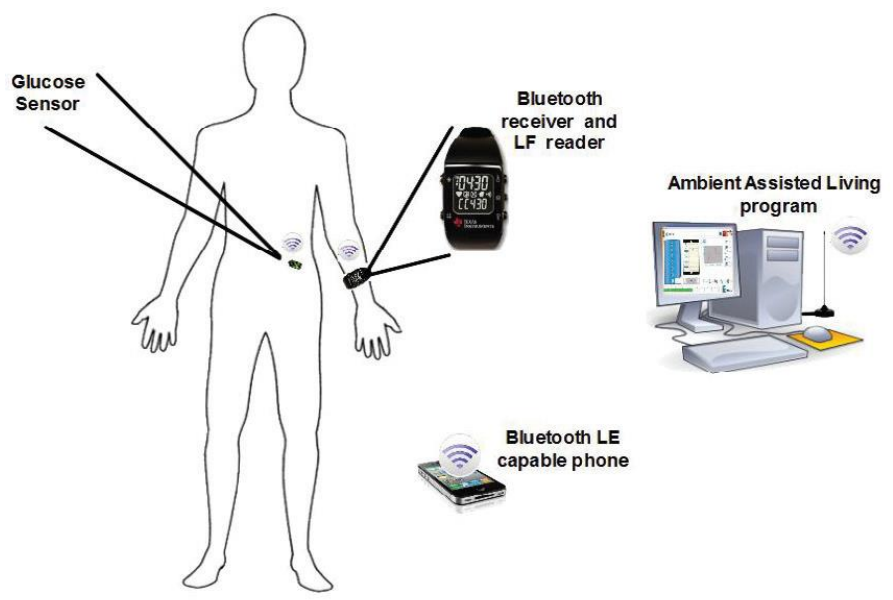

Figura 3 - Sistema Global Ambient Assisted Living.

Além de fornecer informações, como os níveis de açúcar no sangue, esse sistema possibilita a conexão para qualquer dispositivo via bluetooth. Essa característica é importante, pois pode-se manter os dados gravados em compartilhamento com os médicos ou até agentes de saúde estabelecendo uma comunicação que possa ajudar a melhorar o trabalho com os diabéticos.

\section{Análise comparativa dos sistemas.}

O Quadro 1 resume as características de cada sistema embarcado que possibilitam a coleta e exibição de dados glicêmicos através de seus aspectos de hardware e funcionalidades. 
Quadro 1. Caraterísticas comparativas.

\begin{tabular}{|c|c|c|c|}
\hline Características & $\begin{array}{c}\text { LATHA et al. } \\
\mathbf{( 2 0 1 2 )}\end{array}$ & $\begin{array}{c}\text { MESHRAM et al. } \\
\mathbf{( 2 0 1 5 )}\end{array}$ & SILVA et al. (2012) \\
\hline Medidor de glicose & Amperométrico & Espectroscopia NIR & Detecção osmótica \\
\hline MCU & PIC18F4520 & Não especificado & CC2540 \\
\hline $\begin{array}{c}\text { Complexidade de } \\
\text { Utilização }\end{array}$ & Baixa & Baixa & Baixa \\
\hline $\begin{array}{c}\text { Exibição dos Dados } \\
\text { Armazenamento de } \\
\text { Dados }\end{array}$ & $\begin{array}{c}\text { Display LM16200 } \\
(16 x 2 \text { caracteres })\end{array}$ & Smartphone & $\begin{array}{c}\text { Computador ou } \\
\text { Smartphone }\end{array}$ \\
\hline $\begin{array}{c}\text { Transmissão de } \\
\text { Dados }\end{array}$ & Sim & Sim & Sim \\
\hline Custo & Baixo & GSM & Bluetooth \\
\hline
\end{tabular}

\subsection{Medidor de glicose.}

O sistema proposto pelos [Latha et al. 2012] exige que o indivíduo realize um pequeno furo no dedo da mão, extrair uma gota de sangue, depositá-la numa fita própria e introduzir a fita no aparelho, em outras palavras, um método invasivo.

No caso do sistema de [Meshram et al. 2015] é utilizado o método óptico baseado em infravermelho, também conhecida como método de Espectroscopia NIR. Portanto, um sistema não-invasivo.

Em [Silva et al. 2012], o sensor utilizado para os testes foi o medidor baseado na detecção osmótica de glicose. Essa nova tecnologia de sensoriamento visa conduzir medições sem dor para o paciente e possui boa capacidade de conservação, bem como adaptabilidade à miniaturização por microfabricação tornando 0 sistema de monitoramento pequeno o suficiente para permitir a implantação subcutânea sob a pele apenas por injeção. Assim, não haverá exigência para cirurgia [Leal et al. 2011].

O Quadro 2 resume as vantagens e desvantagens do método de medição de glicose utilizado por cada sistema revisado.

Quadro 2. Vantagens e desvantagens dos métodos utilizados nos sistemas revisados.

\begin{tabular}{|c|c|c|}
\hline Sistema & Vantagens & Desvantagens \\
\hline Latha et al. (2012) & $\begin{array}{c}\text { Fácil manuseio } \\
\text { Baixo custo }\end{array}$ & $\begin{array}{c}\text { Requer amostra de sangue } \\
\text { Necessidade de fichas de teste } \\
\text { Pode ser necessário um volume } \\
\text { maior de sangue no teste. } \\
\text { Erros acurácia }\end{array}$ \\
$\begin{array}{c}\text { Meshram, et al. } \\
(2015)\end{array}$ & $\begin{array}{c}\text { Método não-invasivo } \\
\text { Medições satisfatórias } \\
\text { Não requer amostra de sangue }\end{array}$ & $\begin{array}{c}\text { Mais suscetível à interferência } \\
\text { Maior dificuldade de calibração }\end{array}$ \\
\hline Silva et al. (2012) & $\begin{array}{c}\text { Não precisa de cirurgia } \\
\text { Medições mais eficientes } \\
\text { Maior nível de conservação }\end{array}$ & $\begin{array}{c}\text { Requer amostra de sangue } \\
\text { Método invasivo }\end{array}$ \\
\hline
\end{tabular}




\subsection{Microcontrolador}

[Latha et al. 2012] emprega o MCU PIC18F4520 para ligar todos os componentes no medidor de glicemia. Esse modelo PIC18 pertence à uma família de microcontroladores 8 bits que combina o melhor desempenho com menor custo de aquisição entre as opções disponíveis do PIC.

Ele utiliza a linguagem $\mathrm{C}$ na programação de firmware, contém recurso de Interrupção, Conversor A/D e memória do tipo FLASH, características que facilitam o desenvolvimento de projetos embarcados. Exige que o programador tenha conhecimentos aprofundados de arquitetura de computadores, eletrônica e programação avançada, ou seja, voltada para desenvolvedores da área.

[Meshram et al. 2015] não especifica o MCU utilizado, mas apenas que o protótipo desenvolvido precisa de um circuito baseado em microcontrolador para converter os valores em valores de glicose no sangue correspondentes, que são então exibidos no computador através de GSM. Logo, conclui-se que qualquer modelo de microcontrolador com características mínimas para o funcionamento do sistema pode ser utilizado.

[Silva et al. 2012] utiliza o CC2540 de tamanho pequeno que pode ser acoplado um receptor bluetooth como vários outros componentes. Esse dispositivo consome extremamente pouca energia, oferece uma taxa de transmissão de $1 \mathrm{Mbps}, 8 \mathrm{kB}$ de memória RAM e $128 \mathrm{kB}$ ou $256 \mathrm{kB}$ de memória flash, dependendo da versão. A desvantagem está no fato de apresentar um menor desempenho de processamento comparado às plataformas microcontroladas como PIC e Arduino [Maio 2014].

\subsection{Complexidade de utilização}

A versão desenvolvida pelos [Silva et al. 2012] apresenta o menor nível de complexidade de utilização porque é o mais robusto entre os sistemas revisados capaz de transmitir as informações de forma constante para qualquer dispositivo via conexão bluetooth, após a implantação na pele. Não necessita de treinamento prévio.

Com o segundo menor nível de complexidade, o sistema proposto pelos [Latha, et al. 2012] não necessita de implantação ou cirurgia e exige pouco treinamento de manuseio.

O sistema desenvolvido pelos [Meshram et al. 2015] é o mais complexo devido aos problemas de interferências e instabilidade do medidor utilizado. A estabilidade depende de um acoplamento óptico constante à pele, que é difícil de manter a menos que o paciente esteja deitado e imóvel.

\subsection{Exibição dos Dados}

[Latha et al. 2012] demonstra em seu projeto o display de cristal líquido de modelo LM16200 (16x2) ligado ao microcontrolador PIC18F4520. O protótipo de [Meshram et al. 2015] exibem os dados em smartphone ou computador através da tecnologia de modem GSM e assim poder interagir através de aplicativos incorporados ao sistema. Já 
em [Silva et al. 2012], a exibição de dados acontece por conexão bluetooth com um computador, smartphone ou outro dispositivo que possa conectar-se com o sistema.

\subsection{Armazenamento de Dados}

Em [Meshram et al. 2015] especifica o armazenamento de dados coletados em servidor FTP, que através da rede conectada à Internet pode ter acesso aos dados do usuário com um disco ou servidor de arquivos de protocolo de transferência de arquivos. [Silva et al. 2012] não detalha o tipo de armazenamento via servidores, mas envia dados a dispositivos, como telefones e computadores que podem armazenar esses dados. Em [Latha et al. 2012], não há armazenamento dos dados coletados.

\subsection{Transmissão de Dados}

O protótipo de [Meshram, et al. 2015] descreve a junção de um modem GSM, podendo ser utilizado o GPRS, que permite conexão com a internet de maneira flexível ao analisar e diagnosticar valores de glicemia, enquanto que a transmissão de dados do protótipo de [Silva et al. 2012] é realizada via bluetooth com qualquer dispositivo com capacidade de conexão e exibição dos dados. Em [Latha et al. 2012], não há transmissão dos dados coletados.

\subsection{Custo}

Silva, et, al, (2012) relata o sistema como um meio de reduzir os custos no cuidado à saúde, que é um fator importante para melhorar a qualidade de saúde visando a obtenção de tecnologia de baixo custo para as pessoas. O preço do CC2540 apresenta baixo custo e boa capacidade de conservação que ajuda a reduzir os custos de manutenção. Não há custos de utilização.

Por ser um sistema simples e que não utiliza tecnologias de transmissão e armazenamento de dados, o sistema proposto por [Latha et al. 2012] apresenta custo de projeto relativamente baixo em relação a outros tipos de dispositivos comercializado que fazem verificação de açúcar no sangue. O custo de aquisição basicamente é referente ao uso do PIC18F4520. Já o custo de uso é alto devido a necessidade de utilizar tiras e fitas de testes de glicemia.

O protótipo de [Meshram et al. 2015] necessita do modem GSM e de um MCU que possa se comunicar com ele. Independente da escolha do MCU, o sistema apresenta um custo alto de instalação e manutenção em comparação aos demais.

\section{Considerações finais}

No que se refere ao método de glicemia utilizado, o sistema desenvolvido pelos Silva, et, al (2012) apresenta melhor alternativa, pois não precisa de cirurgia (o sensor é injetado na pele), apresenta medições mais eficientes e maior durabilidade. Em relação à complexidade de utilização eles apresentam um sistema com menor nível de complexidade de utilização porque é o mais robusto entre os sistemas revisados capaz de transmitir as informações de forma constante para qualquer dispositivo via conexão bluetooth. Também responde melhor aos requisitos de preço final do produto e consumo de energia por utilizar um MCU com processamento suficiente para a execução e controle do medidor de glicose. 
No critério de exibição de dados, o protótipo de [Latha et al. 2012] é o mais simples e limitado porque utiliza apenas um display de cristal líquido de modelo LM16200 (16x2).

Sobre a capacidade de armazenamento dos dados coletados, [Meshram et al. 2015] especifica o armazenamento de dados coletados em servidor FTP, que através da Internet pode ter acesso aos dados do usuário com um disco ou servidor. Na função de transmissão de dados, o protótipo de [Meshram et al. 2015] descreve a junção de um modem GSM que permite conexão com a internet de maneira flexível ao analisar e diagnosticar valores de glicemia.

Como trabalho futuro é pretendido propor um sistema embarcado que combina as vantagens de cada sistema revisado e que haja validações envolvendo grupo de pessoas ou espécies de animais, visto que isto não foi relatado nos três trabalhos.

\section{Referências}

Chaves, Filipe Ribeiro et al. (2018). "Bengala e capacete ultrassônico para detecção de obstáculos para deficientes visuais". Encontro Unificado de Computação (ENUCOMPI).

Heintzman, N. D. (2016). "A digital ecosystem of diabetes data and technology: services, systems, and tools enabled by wearables, sensors, and apps. Journal of diabetes science and technology", 10(1), 35-41.

Latha, N. A., Murthy, B. R., and Sunitha, U. (2012). "Design and development of A microcontroller based system for the measurement of blood glucose. Measurement", 2(5).

Maio, A. J. D. F. (2014). "Bluetooth Low Energy para monitorização da postura no ciclismo" (Doctoral dissertation).

Meshram, N. D., and Dahikar, P. B. (2015, January). "Microcontroller Based Instrument for Semi-Invasive Blood Glucose Measurement". In International Symposium on Ultrasonics (Vol. 22, No. 24).

Oliveira Junior, M., and Duarte, R. D. O. (2011). Apostila sobre introdução ao projeto com microcontroladores e programação de periféricos. Departamento de Engenharia Eletrônica-Escola de Engenharia-Universidade Federal de Minas Gerais.

Penido, É. D. C. C., and Trindade, R. S. (2013). "Microcontroladores". Ouro Preto: IFMG.

Silva, S., Martins, H., Valente, A., and Soares, S. (2012). "A bluetooth approach to diabetes sensing on ambient assisted living systems". Procedia Computer Science, 14, 181-188.

SBD, SOCIEDADE BRASILEIRA DE DIABETES. (2015) "Gerenciamento eletrônico do diabetes: usando a tecnologia para melhor controle metabólico do diabetes". Disponível em: <https://www.diabetes.org.br/profissionais/diabetes-tipo-1/83gerenciamento-eletronico-do-diabetes-usando-a-tecnologia-para-melhor-controlemetabolico-do-diabetes>. Acesso em: 29 de maio de 2020.

Wolf, M. (2012). Computers as components: principles of embedded computing system design. Elsevier. 